DOI: 10.12957/demetra.2015.14870

\title{
Análise da produção científica do Curso de Nutrição da Universidade Federal de Mato Grosso
}

\section{Analisys of sientific production of Nutrition Course of Federal University of Mato Grosso}

\author{
Tarsis de Mattos Maial \\ Lorena Barbosa Fonsecal \\ Emanuele Batistela' \\ Raquel Azambuja Reinaldo? \\ Luana Pucci de Lima' \\ Maria Aparecida de Lima Lopes' \\ 1 Universidade Federal de Mato Grosso, \\ Departamento de Alimentos e Nutrição, \\ Faculdade de Nutrição. Cuiabá-MT, Brasil. \\ Correspondência / Correspondence \\ Lorena Barbosa Fonseca \\ Universidade Federal de Mato Grosso, \\ Departamento de Alimentos e Nutrição, \\ Faculdade de Nutrição \\ Av. Fernando Corrêa da Costa, n ${ }^{0} 2367$ - 78060 - \\ 900 Cuiabá-MT, Brasil \\ E-mail: lorenanutrica0@gmail.com
}

\section{Resumo}

O curso de Nutrição da Universidade Federal de Mato Grosso foi criado em 1978 e implantado a partir de 1979, tendo crescido de forma gradual e lenta em seus primeiros anos. O objetivo deste estudo foi avaliar a produção científica do curso ao longo de trinta anos, desde sua criação. Os dados foram coletados a partir da busca ao currículo Lattes dos docentes ativos e inativos do curso. Identificou-se aumento expressivo do total de publicações no decorrer do período investigado (1981-2011). A maioria (52,6\%) constituiu-se de resumos científicos publicados em anais de eventos, sendo o período de 2001 a 2011 o de maior produção científica, seja por diversidade de tipologia, seja por quantitativo da produção. Os três campos temáticos predominantes no período estudado relacionam-se à natureza biológica das pesquisas em Nutrição, sendo eles a Nutrição Básica e Experimental, seguido pela Ciência e Tecnologia de Alimentos e Nutrição Clínica, este último dividindo espaço com o campo da Avaliação Nutricional de Populações. Embora inserido em contexto nacional, os resultados encontrados limitam-se ao curso, não sendo possível extrapolá-los nem fazer conclusões sobre o assunto, uma vez que são parte da construção histórica do curso de Nutrição da UFMT. Sugere-se que novos estudos sejam feitos considerando o pequeno número de estudos atuais, da análise da produção científica e caminho das publicações no cenário nacional.

Palavras-chave: Produção Científica. Nutrição e Saúde. Publicações. 


\section{Abstract}

The Nutrition course at the Federal University of Mato Grosso, Brazil, was created in 1978 and implemented from 1979, growing gradually and slowly in its early years. This study aimed to evaluate the scientific production over the course of thirty years, since its creation. Data were collected online from Lattes curriculum, on active and retired teachers of the course. It identified significant increase in the total number of publications during the investigated period (1981-2011). Most (52.6\%) consisted of abstracts published in conference proceedings, and the period 2001-2011 showed the highest scientific production, either by type of diversity, either by quantity of production. The three thematic areas prevalent during the study period relate to the biological nature of research in nutrition, and they Basic and Experimental Nutrition, followed by Food Science and Technology and Clinical Nutrition, the latter sharing space with the field of Nutritional Assessment of Populations. Although inserted in the national context, the results are limited to the course, it is not possible to extrapolate them or make conclusions about them, since they are part of the historical construction of the UFMT Nutrition course. Further studies should be made considering the small number of current studies, the analysis of scientific literature and way of publications on the national context.

Key words: Scientific Production. Health and Nutrition. Publications.

\section{Introdução}

O campo da Alimentação e Nutrição ocupa lugar de destaque nas agendas nacionais e internacionais especialmente voltadas ao desenvolvimento humano e à sustentabilidade ecológica do planeta. No Brasil, a agenda pública é materializada pela atual Política Nacional de Alimentação e Nutrição. ${ }^{1,2}$ Esta, por sua vez, tem como principal agente do referido campo o profissional nutricionista. A Universidade Federal de Mato Grosso (UFMT) vem, desde a década de 1970, quando da criação do curso, contribuindo para a formação de profissionais nutricionistas críticos, reflexivos e de elevado nível técnico. 
O curso de Nutrição, criado em 1978 e implantado a partir de 1979, assim como os demais cursos e instituições públicas de ensino de Nutrição do país, cresceu de forma gradual e lenta em seus primeiros anos. Este fato se explica pela não capacitação docente e técnica, em nível de pós-graduação lato sensu e, sobretudo, stricto sensu. Contudo, nos últimos trinta anos, houve um estímulo progressivo à produção científica no Brasil que poderia ser explicado por vários fatores, dentre os quais o crescimento dos programas de pós-graduação e a conseguinte capacitação docente e técnica em nível de mestrado e doutorado, o sistema de avaliação da pós-graduação, o financiamento e a valorização da qualidade dos curricula vitae. ${ }^{3,4}$

A produção científica brasileira tem crescido significativamente ao longo das últimas décadas. A média anual de trabalhos indexados em uma importante base bibliográfica internacional e cujos autores ou coautores eram brasileiros, aumentou de menos de mil na década de 80 para quase 6.000 no ano de 2000. ${ }^{5}$ Segundo dados da Academia Brasileira de Ciências, ${ }^{6}$ em 2009 o Brasil produziu 32 mil artigos em publicações científicas, equivalente a $54 \%$ da produção latinoamericana e 2,7\% da mundial. Atualmente, o país ocupa o 13 o lugar no ranking dos países com maior produção científica no mundo. ${ }^{7}$

O desenvolvimento científico ocorrido nas últimas décadas foi significativamente influenciado por temas de grande impacto para a humanidade, como a conservação do meio ambiente, os recursos alimentares e energéticos, a saúde, o transporte, os meios de comunicação, bem como as condições de melhoria da qualidade de vida do ser humano, em geral. ${ }^{4}$ A ciência da Nutrição, enquanto campo multidisciplinar, está inserida nesse contexto, sendo sua pesquisa (com)partilhada por distintas ciências e profissionais. ${ }^{8} \mathrm{O}$ potencial de crescimento da área pode ser ilustrado pelas recentes discussões sobre o fortalecimento das pós-graduações, pelo incremento do número de grupos de pesquisas e de publicações na área, pelas discussões acerca dos campos científicos que envolvem o campo da Nutrição e, recentemente, pela criação da área de Nutrição na Capes. ${ }^{2,9,10}$

Diante de tais circunstâncias, é de se esperar que a produção científica do curso de Nutrição dessa instituição tenha se apropriado das melhorias no campo e que tenha ocorrido aumento quantitativo e qualitativo em sua produção, em consonância com o cenário nacional. No entanto, esses avanços precisam ser divulgados e analisados.

Moreno \& Márdero Arellano ${ }^{11}$ ressaltam a importância da publicação científica dentro do ciclo do conhecimento científico, que compreende a produção, a comunicação e a aplicação do conhecimento gerado. Nesse ciclo, a publicação científica exerce função essencial na medida em que a divulgação dos resultados de pesquisa auxilia na construção do conhecimento. O conhecimento, por sua vez, para ser consolidado, depende da disseminação e transferência das informações, e precisa ser analisado em um processo interativo de discussão e aprovação pelos pares, o que garante confiabilidade à pesquisa. 
Para Mueller, ${ }^{12}$ a comunicação é ato inerente a toda pesquisa científica e se realiza segundo práticas estabelecidas, podendo ser registrada em veículos formais por meio de livros, periódicos ou meios eletrônicos - a literatura científica. Nas últimas décadas, o acesso a tal literatura teve seus mecanismos de divulgação ampliados, com a evolução da rede e de softwares abertos. ${ }^{11}$ Também o portal da Capes, por meio do banco de teses - e de periódicos de livre acesso -, assim como o portal do CNPq, via Plataforma Lattes, constituem importantes ferramentas para o reconhecimento dos grupos de pesquisas certificados existentes ${ }^{9}$ e de suas produções.

Reconhecendo a complexidade do ciclo da produção científica, o que se propõe para o presente trabalho, seguindo as discussões levantadas por Kac et al., ${ }^{9}$ é o estudo da produção científica do curso de Nutrição da Universidade Federal de Mato Grosso - ou seja, a realização de um autorreconhecimento de sua produção. Debruçar-se sobre o que foi produzido permitirá planejar as futuras investigações e investimentos, além de promover o crescimento e a contínua qualificação da produção científica do curso de Nutrição da UFMT, o que refletirá na qualidade da formação da graduação e pós-graduação.

\section{Material e métodos}

Trata-se de estudo retrospectivo, de caráter descritivo, da produção científica do curso de Nutrição da UFMT no período de 1981 a 2011. Para isso realizou-se, junto a instâncias administrativas da faculdade, um levantamento, no período considerado, dos docentes (ativos e não ativos) do Curso de Nutrição/FANUT/UFMT.

Em seguida, procedeu-se ao levantamento da produção científica de cada docente. Considerouse exclusivamente a produção registrada por cada docente no currículo da Plataforma Lattes. Para isso, foi realizada a busca de cada currículo durante o período de outubro de 2013 a fevereiro de 2014. Finalmente, foram definidos como produção científica de interesse para o presente estudo: artigos completos publicados em periódicos científicos; teses e dissertações de autoria, sob orientação e co-orientação dos referidos docentes; livros e capítulos de livros; trabalhos completos publicados em anais de eventos e resumos publicados em anais de eventos.

Em seguida, classificou-se a produção científica em 11 campos temáticos da Nutrição, adaptada de Vasconcelos, ${ }^{13}$ como segue:

1- Nutrição Básica e Experimental: ensaios biológico-experimentais, estudos com animais, metabolismo;

2- Ciência e Tecnologia de Alimentos: análise químico-bromatológica, composição químiconutricional, valor calórico, teor mineral e vitamínico dos alimentos, microbiologia de alimentos; 
3- Nutrição Clínica (incluindo fisiologia, patologia e dietoterapia): estudos com humanos;

4- Nutrição e Dietética: técnica dietética, culinária, dietética propriamente dita;

5- Avaliação Nutricional de Populações: uso de métodos antropométricos, clínicos, laboratoriais, dietéticos, demográficos e socioeconômicos para a realização de diagnósticos nutricionais de comunidades;

6- Política e Planejamento em Saúde, Alimentação e Nutrição: trata de políticas, programas de alimentação e nutrição e SAN;

7- Epidemiologia: estudo das doenças em determinada população;

8- Determinantes do Estado Nutricional e de Saúde de Populações: gênese, reprodução e consequências da problemática alimentar, nutricional e de saúde no Brasil;

9- Educação Alimentar e Nutricional: escolhas alimentares, comportamento alimentar, promoção da saúde e de alimentação saudável;

10- Educação no Ensino Superior: formação de profissionais da saúde, avaliação, acesso ao ensino superior, ensino-aprendizagem;

11- Alimentação Coletiva: boas práticas, planejamento e administração de serviços de alimentação, etc.;

12- Outros.

Para os artigos científicos, foram ainda definidas as seguintes categorias analíticas: tipo de estudo, método e sujeitos.

Os dados coletados foram digitados em um banco de dados elaborado no software Microsoft Office Excel $2007^{\circledR}$. As informações foram analisadas de forma descritiva, por meio de medidas de frequências (relativas e absolutas).

Com o intuito de subsidiar e contextualizar as discussões, realizou-se um resgate histórico do Curso de Nutrição na UFMT por meio de pesquisa documental e entrevista semiestruturada com gestores da Faculdade de Nutrição e a coordenação do curso.

\section{Resultados e discussão}

O curso de Nutrição da UFMT, assim como vários outros cursos superiores de graduação, foi implantado em um momento histórico posterior à reforma universitária de 1968, que determinou a abertura de novos cursos em todas as áreas de conhecimento, sobretudo naquelas que contavam 
com menor número de cursos de graduação e, também, por incentivo do II PRONAN (Programa Nacional de Alimentação e Nutrição), em sua diretriz específica de formação de recursos humanos. ${ }^{13,14}$

Criado, pois, dentro da lógica organizacional e de funcionamento estabelecida pela reforma universitária, o curso assumiu a pesquisa, desde sua criação, enquanto parte dos objetivos da universidade, conforme estabelecido na Lei no 5.540/1968, ao considerar a pesquisa como item indissociável do ensino superior nas universidades..$^{15}$

Nesse sentido, ao se analisar a produção científica do curso de Nutrição da UFMT em termos quantitativos, foi possível identificar aumento expressivo do total de publicações no decorrer do período investigado (1981-2011). Ainda que a pesquisa estivesse expressamente ligada ao âmbito da universidade, reconhece-se que na prática, como era de se esperar, a produção científica foi mais lenta na década seguinte à criação do curso (década de 1980) e intensificou-se a partir da década de 1990, conforme ilustra o gráfico 1. O aumento gradativo da produção científica ao longo do período coincide com o desenvolvimento do curso e com a própria qualificação do corpo docente, que veio a concluir sua pós-graduação stricto sensu anos depois de ingressarem na carreira.

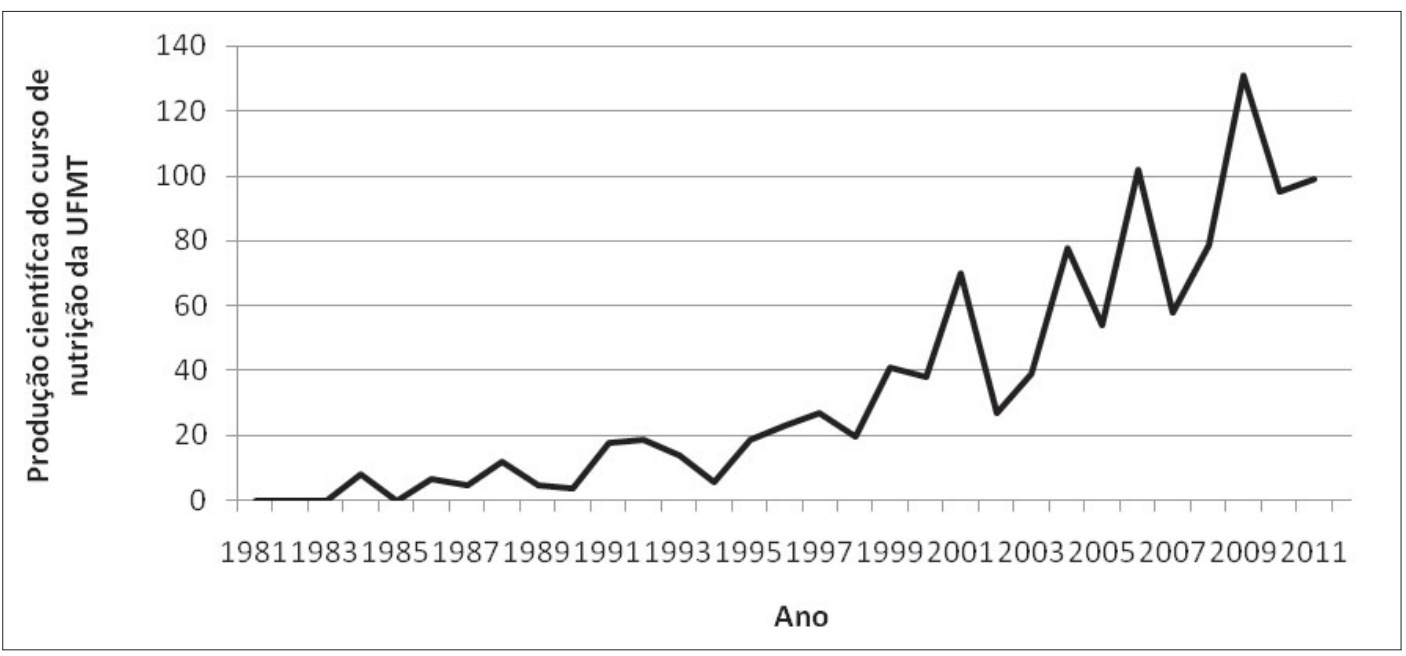

Gráfico 1. Produção científica do Curso de Nutrição da UFMT no período de 1981 a 2011. 
De acordo com Kac, ${ }^{2}$ os primeiros cursos de pós-graduação em Nutrição lato sensu (especialização) tiveram início na década de 1960, e os cursos de pós-graduação em Nutrição stricto sensu (mestrado), no começo da década de 1970. No período de 1971 a 1991, estavam registrados na Capes quatro cursos de mestrado e três de doutorado, concentrados na Região Sudeste, e apenas um situava-se na Região Nordeste do Brasil. ${ }^{2}$

Interessante ressaltar que a formação em pós-graduação para nutricionistas, em razão da natureza interdisciplinar dos estudos desenvolvidos na área de Nutrição, amplia-se para diferentes áreas do conhecimento, permitindo que esses profissionais busquem sua qualificação nos mais diferentes programas. Entretanto, a oferta de cursos de pós-graduação em Nutrição fora do eixo sul-sudeste pode ser considerada recente, tendo ocorrido a partir dos anos 2000, juntamente com a ampliação do número de cursos de pós-graduação, tendo avançado especialmente com a criação da área de Nutrição na Capes, em 2006. ${ }^{9}$

Se considerarmos o conjunto das publicações em estudo, percebe-se que a maioria $(52,6 \%)$ das publicações constituiu-se de resumos científicos publicados em anais de eventos, segundo o gráfico 2. E o gráfico 3 ilustra a predominância dos resumos científicos, por intervalo de dez anos, sendo o período de 2001 a 2011 o de maior produçãocientífica, seja por diversidade de tipologia, ou por quantitativo da produção. Esse período coincide com a criação, na estrutura da Faculdade de Nutrição, em 2006, do programa de pós-graduação stricto sensu, reforçando o papel da pósgraduação no desenvolvimento da pesquisa dentro da instituição.

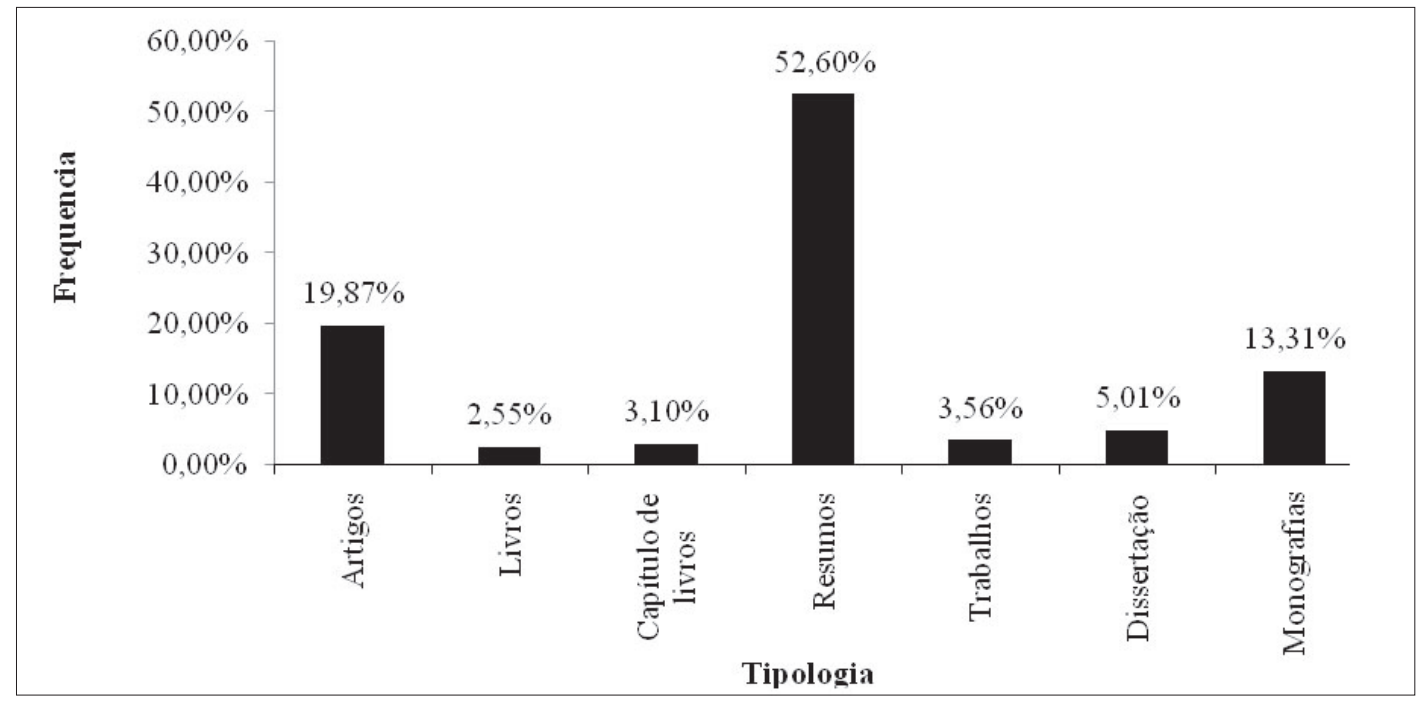

Gráfico 2. Frequência da produção científica do Curso de Nutrição da UFMT no período de 1981 a 2011, segundo tipologia. 


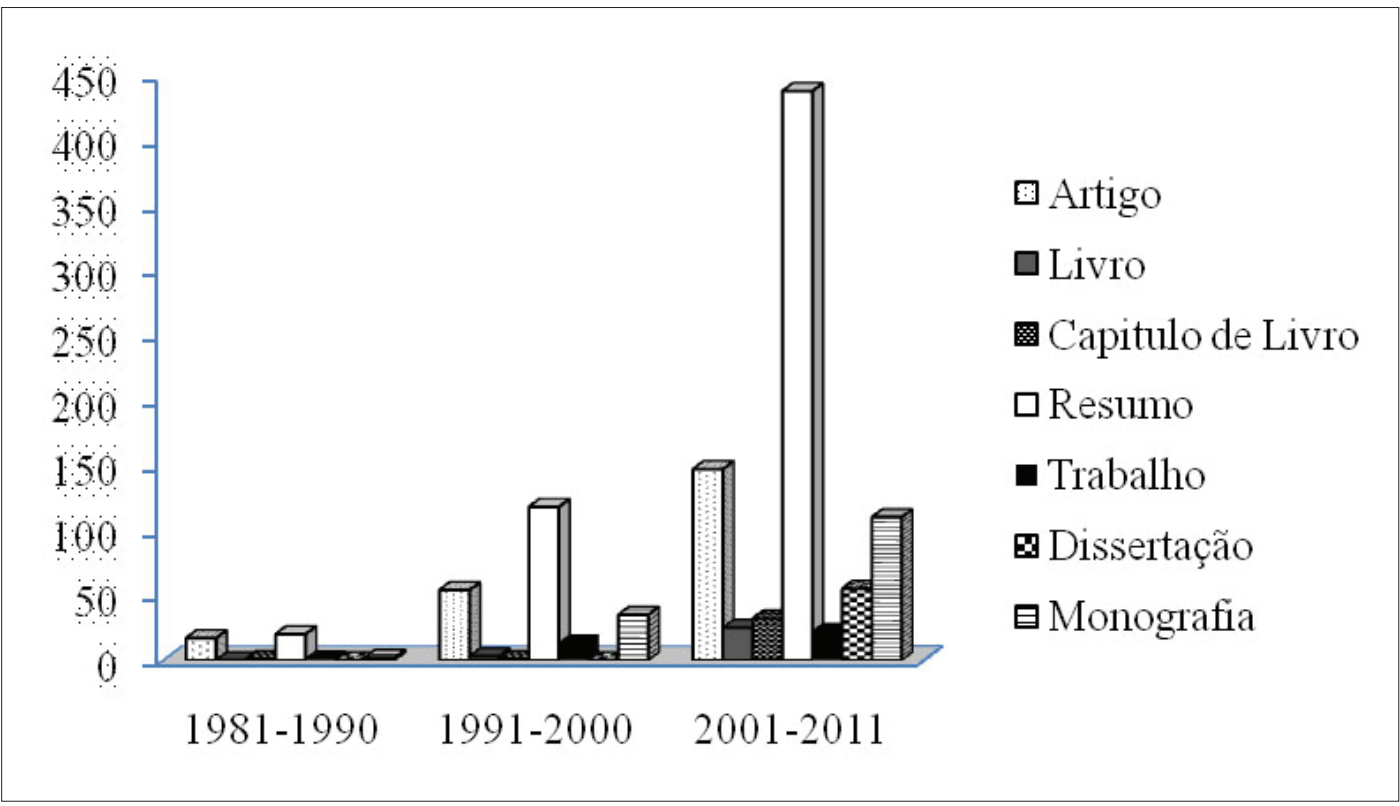

Gráfico 3. Produção científica do Curso de Nutrição da UFMT, segundo a tipologia, no período de 1981 a 2011, em intervalo de dez anos.

Ao analisarmos a produção científica segundo campos temáticos, percebe-se que os três campos predominantes no período estudado relacionam-se a natureza biológica das pesquisas em Nutrição, sendo eles a Nutrição Básica e Experimental, seguido pela Ciência e Tecnologia de Alimentos e Nutrição Clínica, este último dividindo espaço com o campo da Avaliação Nutricional de Populações (gráfico 4). Esse resultado coincide com os achados de Vasconcelos em trabalho publicado em 1999, ainda que mais de vinte anos tenham se passado desde a análise conduzida pelo autor sobre a produção científica em Nutrição em periódicos da área. ${ }^{16}$ 


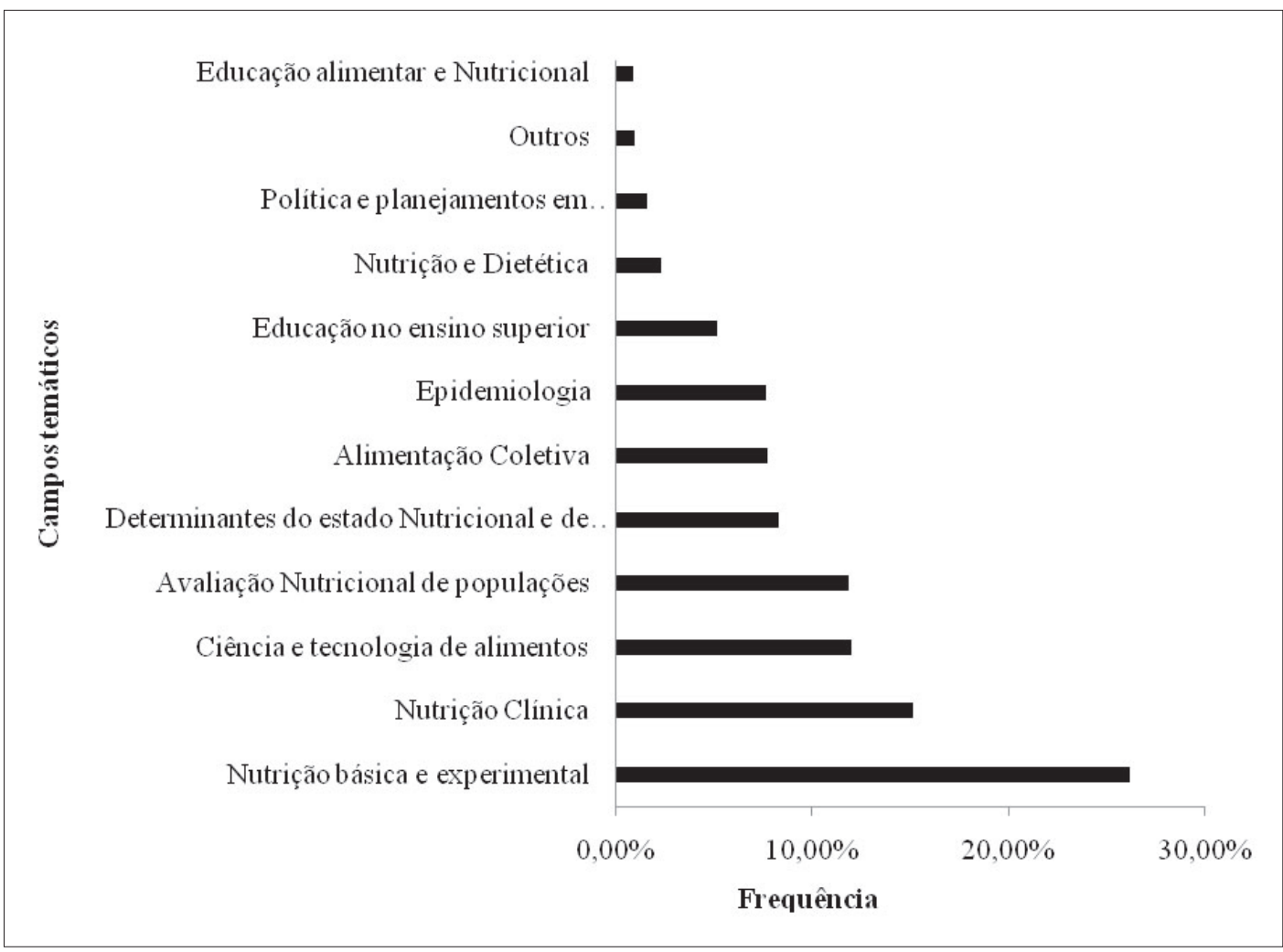

Gráfico 4. Distribuição da produção científica do Curso de Nutrição da UFMT segundo campos temáticos, no período de 1981 a 2011.

Embora não permita generalizações, como assume o próprio autor, ${ }^{16}$ pode-se observar que, quanto à natureza da pesquisa, comparando com resultados encontrados no presente estudo, os campos temáticos da perspectiva biológica, no que concerne à produção científica do curso de Nutrição da UFMT, nasceram juntamente com o curso. Foram influenciados também por aspectos histórico-conjunturais do período e se consolidaram e cresceram ao longo do tempo, dividindo espaço com outros campos temáticos, em especial o de Avaliação Nutricional de Populações, conforme ilustra o gráfico 5 , ao distribuir a frequência das publicações por campos temáticos em intervalos de dez anos. 


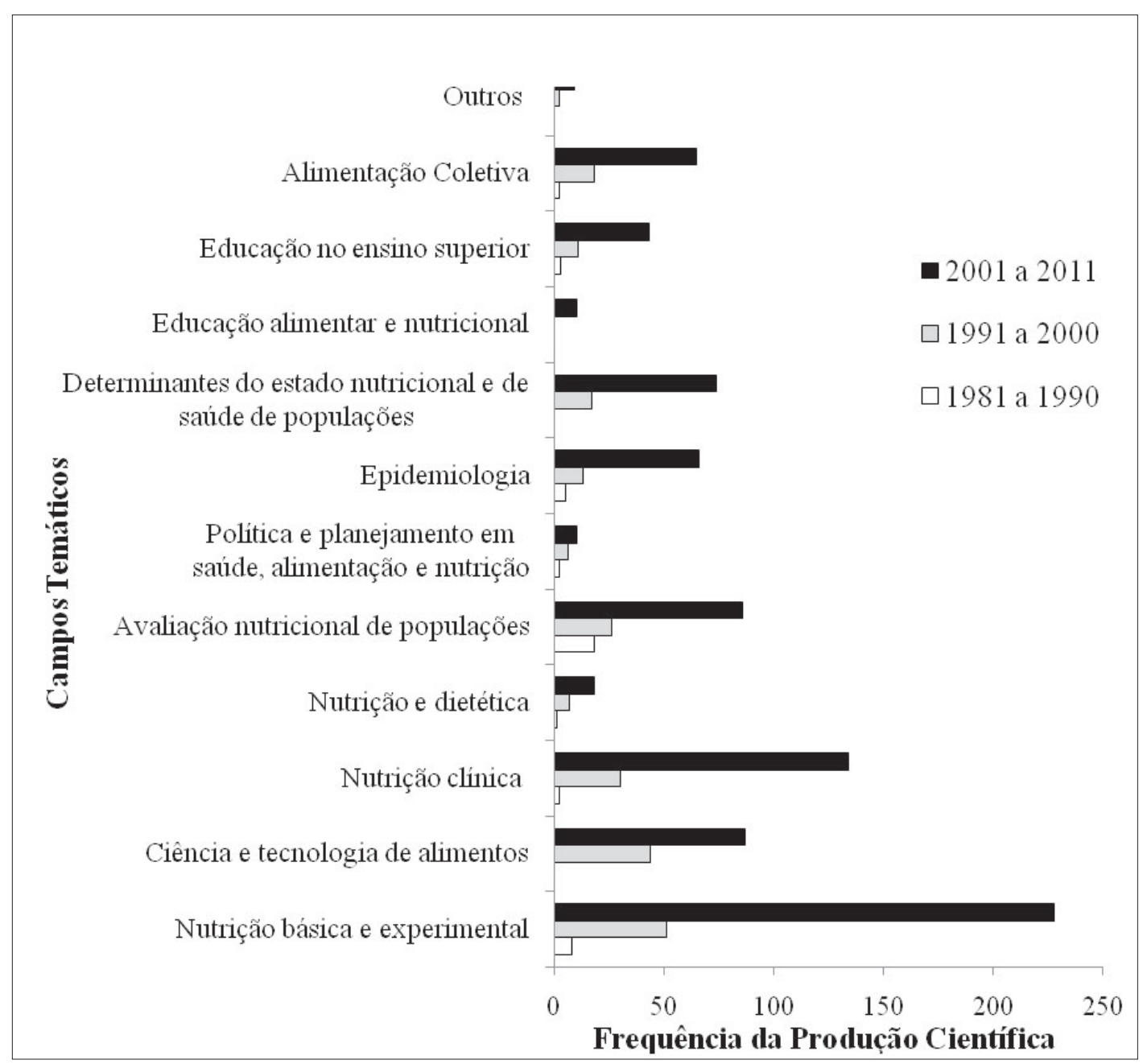

Gráfico 5. Produção Científica do Curso de Nutrição da UFMT segundo campos temáticos, no período de 1981 a 2011, em intervalo de dez anos. 
A separação entre a perspectiva biológica e a perspectiva social ocorre também nas discussões sobre a própria formação profissional da categoria, em âmbito nacional. Costa, ${ }^{17} \mathrm{em}$ trabalho que investigou os estudos e eventos que discutiram a formação do nutricionista desde seu surgimento até a década de 1990, retrata que "uma constatação sempre presente nos eventos e estudos sobre a formação do nutricionista é a existência de um 'hiato entre o biológico e o social' nos currículos" (p. 17), apontando a necessidade de mudança na formação no sentido de formar um profissional engajado com as transformações da sociedade. Também Bosi,${ }^{18} \mathrm{em}$ seu estudo, ao investigar os discursos sobre a Nutrição enquanto ciência, política e o discurso institucional do aparelho formador, encontra que a estrutura curricular dos cursos de Nutrição não só privilegiava a visão biológica da Nutrição como influenciava a própria difusão do saber científico em Nutrição no meio acadêmico.

Importa reforçar que tal separação entre o biológico e social, e, como assume Costa, ${ }^{17}$ também o técnico e político, não foram vivenciados apenas pelos cursos de graduação em Nutrição, mas por todos os cursos da área da saúde, tendo em vista a predominância do modelo biomédico de formação. ${ }^{19}$

Ainda analisando os resultados encontrados no gráfico 5, chama a atenção a escassez de pesquisas no campo temático da Educação Alimentar e Nutricional, e também na Alimentação Coletiva. Pode-se inferir, pelo contexto histórico da época, quando a fome e a desnutrição, principais problemas nutricionais, foram associadas à baixa renda da população, que a educação nutricional foi assumida como desnecessária, observando-se no cenário nacional poucas publicações na área, ${ }^{20}$ o mesmo ocorrendo no curso, como observado no período de 1981-1990 (gráfico 5).

Quanto ao campo da Alimentação Coletiva, chama a atenção o fato de que, embora à época o graduado em Nutrição tivesse uma formação eminentemente voltada para o planejamento de cardápios e controle de estoque (atividades da Alimentação Coletiva) e dietoterapia (atividade da Nutrição Clínica), segundo relato da primeira coordenadora do curso, as publicações nas duas áreas não se destacaram até o ano 2000. Especificamente no campo da Alimentação Coletiva, até hoje a produção científica na área, em nível nacional, é considerada insuficiente. ${ }^{21}$

Canella ${ }^{22}$, em estudo que avaliou a produção científica brasileira sobre Nutrição na atenção primária à saúde até o ano de 2011, também observou predomínio de estudos publicados na última década.

Em relação às categorias analíticas, a maioria dos artigos era original, de natureza quantitativa e realizada com seres humanos (tabela 1), seguindo a mesma tendência observada por Canella. ${ }^{22} \mathrm{O}$ predomínio de artigos de natureza quantitativa reflete o que normalmente ocorre nas pesquisas da área da saúde, correspondendo à necessidade de quantificar eventos, causas e efeitos, esquecendose, muitas vezes, da merecida análise da complexidade inerente aos fenômenos relacionados à Alimentação e Nutrição, possível apenas com a realização de pesquisas qualitativas. 
Tabela 1. Classificação dos artigos segundo categorias analíticas (N=218). Cuiabá-MT, 19812011.

Categorias analíticas
Frequência

$\mathrm{N}(\%)$

Tipo de estudo

Original

183

83,94

Revisão

15

06,88

Estudo de caso

1

00,46

Relato de Experiência

3

01,38

Não foi possível identificar

16

07,34

Método

Quantitativo

163

74,77

Qualitativo

7

03,21

Misto

Não se Aplica

1

00,46

28

12,84

Não foi possível identificar

19

08,72

Sujeito

Gestantes

2

00,92

Crianças

20

09,17

Adolescentes

6

02,75

Adultos

18

08,26

Idosos

3

01,38

Profissionais de saúde

2

00,92

Misto

31

14,22

Animais

56

25,69

Não se aplica

60

27,52

Não foi possível identificar

20

09,17 
A pequena produção na área de Nutrição Social - incluindo estudos nos campos temáticos de Avaliação Nutricional de Populações; Política e Planejamento em Saúde e Nutrição; Epidemiologia; Determinantes do Estado Nutricional e de Saúde de Populações; e Educação Alimentar e Nutricional - pode estar relacionada à formação mais voltada, naquela época, para área de Ciência dos Alimentos e Alimentação Coletiva.

Assim como observado neste estudo em relação ao aumento na produção científica ao longo dos anos, nos rankings internacionais observamos ascendência das publicações brasileiras. Entretanto, em uma análise mais crítica, tal ascensão, vista como "prêmio de consolação" ao docente pesquisador por Machado \& Bianchetti, ${ }^{23}$ é fruto de um modelo capital de produção acadêmica, ou produtivismo acadêmico, que muitas vezes acaba por (re)produzir o conhecimento, quando o que se espera é justamente o oposto. ${ }^{24}$

A pesquisa deve permitir ao docente ser o ator principal na sua fala e contribuir com a sociedade. Deve significar o docente como "sujeito", e não como objeto ou coisa dentro de objetivos meramente quantitativos de produção científica. Assim, a busca do saber, da formação do sujeito e transformação social devem ser o eixo principal da investigação acadêmica. ${ }^{24}$ Deste modo, há que se fazer uma avaliação acurada sobre o incremento da produção científica, para não correr o risco de valorar exacerbadamente a quantidade em detrimento da qualidade.

A avaliação da produção de conhecimento em nível institucional, especialmente em países não desenvolvidos, assume papel importante para a hierarquização de prioridades na alocação de recursos humanos e materiais. Ainda, constitui um dos elementos principais para a elaboração e monitoramento de políticas nacionais de ensino e pesquisa, por permitir um diagnóstico situacional da potencialidade acadêmica de uma instituição. ${ }^{25}$ Permite apresentar como o curso vem se desenvolvendo e como tem contribuído para a solução dos problemas relacionados à área de Alimentação e Nutrição e, de certo modo, conforme refere Vanti, ${ }^{26}$ "dignifica o saber".

Merece salientar a complexidade que envolve esse tipo de avaliação, pois segundo Oliveira et al., ${ }^{25}$ a área de Nutrição, embora constitua uma única área de conhecimento, abrange uma diversidade de temas, como resultado das várias contribuições de outros campos da ciência, fazendo com que os avanços em pesquisa sejam de natureza interdisciplinar e multiprofissional.

A análise da produção científica do curso de Nutrição da UFMT nos permitiu identificar o processo de constituição do campo da Nutrição na instituição, e também como as temáticas abordadas foram sendo transformadas ao longo dos trinta anos que foram objeto deste estudo. Embora estudos da área de Avaliação Nutricional de Populações e Nutrição Básica e Experimental tenham se destacado desde o início, na última década observou-se maior expressão de outros campos temáticos. 
Com relação ao alcance da produção, acredita-se que muito do que foi publicado não atingiu a comunidade científica internacional, tendo em conta o idioma de publicação da maioria dos trabalhos.

\section{Considerações finais}

Embora inserido em contexto nacional, os resultados encontrados limitam-se ao curso, não sendo possível extrapolá-los nem fazer conclusões sobre o assunto, uma vez que são parte da construção histórica do curso de Nutrição da UFMT. Apesar das limitações da busca realizada, o objetivo geral deste estudo, de mostrar o crescimento e a tendência das publicações do curso de Nutrição da UFMT nos seus primeiros trinta anos de história, foi atingido, mesmo que, eventualmente, o número de trabalhos publicados (e realizados) possa ter sido maior do que o observado no presente trabalho.

E ainda, o estudo da produção intelectual do curso de Nutrição da UFMT, ao mesmo tempo em que propicia um autorreconhecimento do que foi produzido no período em questão, permite apontar lacunas, avanços, necessidades de investimento e planejamento da produção científica para os anos vindouros. Sugere-se que novos estudos sejam realizados, considerando o pequeno número de estudos atuais, a análise da produção científica e o caminho das publicações no cenário nacional.

\section{Referências}

1. Brasil. Ministério da Saúde. Política Nacional de Alimentação e Nutrição. Brasília: Ministério da Saúde; 2012.

2. Kac G, Proença RPC, Prado SD. A criação da área “nutrição” na Capes. Rev. Nutr. 2011; 24(6):905-916.

3. Velloso J. Mestres e doutores no país: destinos profissionais e políticas de pós-graduação. Cad. Pesquisa 2004; 34(123):583-611.

4. Brasil. Ministério da Educação. Coordenação de Aperfeiçoamento de Pessoal de Nível Superior. Plano Nacional de Pós-graduação - PNPG 2011-2020. Brasília: Capes; 2010.

5. Mugnaini R, Jannuzzi PM, Quoniam L. Indicadores bibliométricos da produção científica brasileira: uma análise a partir da base Pascal. Ci. Inf. 2004; 33(2):123-131.

6. Academia Brasileira de Ciências. Aumento da produção científica brasileira não se reflete em maior número de patentes [Internet]. Rio de Janeiro: ABC; 2011. Acesso em: 28 mar. 2012. Disponível em: http://www.abc.org.br/article.php3?id_article $=1167$ 
7. Coordenação de Aperfeiçoamento de Pessoal de Nível Superior. Presidente da Capes participa de abertura do ano acadêmico do IOC/Fiocruz e assina acordo de cooperação [Internet]. Brasília: Assessoria de Comunicação Social da Capes; 2012. [Acesso em 28 de mar. 2012.]. Disponível em: http://www.capes.gov.br/36-noticias/5278-presidente-da-capes-participa-de-abertura-do-anoacademico-do-iocfiocruz-e-assina-acordo-de-cooperacao

8. Vasconcelos FAG. A ciência da nutrição em trânsito: da nutrição e dietética à nutrigenômica. Rev. Nutr. 2004; 23(6):935-945.

9. Kac G, Fialho E, Santos SMC, Assis AMO. Reflexões do I fórum de coordenadores de programas de pós-graduação em nutrição no Brasil. Rev. Nutr. 2006; 19(6):785-792.

10. Silva JK, Prado SD, Carvalho MCVS, Ornelas TFS, Oliveira PF. Alimentação e cultura como campo científico no Brasil. Physis 2010; 20(2):413-442.

11. Moreno FP, Márdero Arellano MÁ. Publicação científica em arquivos de acesso aberto. Arquivística. net 2005; 1(1):76-86.

12. Mueller SPM. O crescimento da ciência, o comportamento científico e a comunicação científica: algumas reflexões. Rev. Esc. Bibliotecon. UFMG 1995; 24(1):63-84.

13. Vasconcelos FAG, Calado CLA. Profissão nutricionista: 70 anos de história no Brasil. Rev. Nutr. 2011; 24(4): 605-617.

14. Associação Brasileira de Nutrição. Histórico do nutricionista no Brasil. 1939 a 1989. São Paulo: Atheneu; 1991. Coletânea de depoimentos e documentos.

15. Brasil. Lei n ${ }^{\circ}$ 5.540. Fixa normas de organização e funcionamento do ensino superior e sua articulação com a escola média, e dá outras providências. 28 de nov. de 1968. Diário Oficial da União 29 nov. 1968, Seção 1:10369.

16. Vasconcelos FAG. Os Arquivos brasileiros de nutrição: uma revisão sobre produção científica em nutrição no Brasil (1944 a 1968). Cad. Saúde Pública 1999; 15(1):303-316.

17. Costa NMSC. Revisitando os estudos e eventos sobre a formação do nutricionista no Brasil. Rev. Nutr. 1999; 12(1):5-19.

18. Bosi MLM. A face oculta da nutrição: ciência e ideologia. Rio de Janeiro: Editora da Universidade Federal do Rio de Janeiro; 1998.

19. Amorim SP, Moreira H, Carraro TE. A formação de pediatras e nutricionistas: a dimensão humana. Rev. Nutr. 2011; 14(2):111-118.

20. Brasil. Ministério do Desenvolvimento Social e Combate à Fome. Marco de referência de educação alimentar e nutricional para as políticas públicas. Brasília: MDS; 2012.

21. Fonseca KZ, Santana GR. O nutricionista como promotor da saúde em unidades de alimentação e nutrição: dificuldades e desafios do fazer. Enciclopédia Biosfera, Centro Científico Conhecer 2011; 7(13):1466-1476. 
22. Canella DS, Silva ACF, Jaime PC. Produção científica sobre nutrição no âmbito da Atenção Primária à Saúde no Brasil: uma revisão de literatura. Ciênc. Saúde Coletiva 2013; 18(2):297-308.

23. Machado AMN, Bianchetti L. (Des)fetichização do produtivismo acadêmico: desafios para o trabalhador-pesquisador. Revista de Administração de Empresas 2011; 51(3):244-254.

24. Dias ED, Rorato R. O evolucionismo econômico na pós-graduação brasileira: uma análise a partir da ótica da educação. Avaliação: Revista da Avaliação da Educação Superior 2014; 19(1):193-226.

25. Oliveira AC, Dórea JG, Domene SMA. Bibliometria na avaliação da produção científica da área de nutrição registrada no Cibran: período de 1984-1989. Ci. Inf. 1992; 21(3):239-242.

26. Vanti NAP. Da bibliometria à webometria: uma exploração conceitual dos mecanismos utilizados para medir o registro da informação e a difusão do conhecimento. Ci. Inf. 2002; 31(2):152-162.

Recebido: 03/2/2015

Revisado: $27 / 2 / 2015$

Aprovado: $31 / 3 / 2015$ 